\title{
IMPEDANCE AND SINGLE BUNCH INSTABILITY CALCULATIONS FOR THE ILC DAMPING RINGS *
}

\author{
M. Venturini ${ }^{\dagger}$ (LBNL, Berkeley, California), K. Bane, S. Heifets, Z. Li, C. Ng, A. Novokhatski, \\ G. Stupakov, and R. Warnock (SLAC, Menlo Park, California)
}

\section{Abstract}

An important action item for the damping rings of the International Linear Collider (ILC) is to compute the broadband impedance and, from it, the threshold to the microwave instability. We report on the status of our study and provide a preliminary estimate of the instability threshold based on impedance models developed so far.

\section{INTRODUCTION}

In order for the International Linear Collider (ILC) to meet the luminosity target it is essential that properly damped and stable bunches be extracted from the damping rings (DRs). Past experience has taught that instabilities affecting the longitudinal phase space of beams exiting the DRs can result into significant beam quality degradation along the linac. Although a weak instability could perhaps be tolerated, as was the case for the SLC damping rings after replacement of the original vacuum chamber, a desirable approach is to seek to avoid conditions for longitudinal single-bunch instabilities altogether. Characterization of these instabilities, therefore, is critical and indeed has been indicated as a high-priority item in the $\mathrm{R} \& \mathrm{D}$ agenda toward completion of the Engineering Design Report (EDR) as it carries implications regarding specification of the machine components and choice of the lattice parameters.

There are two aspects to this activity: modelling of the relevant sources of the impedance and study of the beam dynamics based on that modelling. The accuracy of the instability estimates depends on that of the impedance modelling, which in turn would require detailed design of the most significant machine components. However, as a complete technical design of these components is not expected to become available until the end of the work period for the EDR, any instability threshold estimates, although approximate, would still be desirable while the DR design is being finalized. To meet this need, the early stage of our study will be based on approximate models of relevant impedance sources derived from the design of components in existing machines (suitably scaled to meet the DRs basic requirements).

A first tentative assessment of longitudinal single-bunch instabilities was already carried out during the baseline configuration studies [1]. Because the main goal at the time was to provide relative ranking of the different lattices under consideration rather than realistic absolute numbers,

\footnotetext{
* Work supported by DOE, Contracts no. DE-AC02-OSCH11231 and DE-AC02-76SF00515.

† mventurini@1bl.gov, kbane@slac.stanford.edu
}

05 Beam Dynamics and Electromagnetic Fields
Table 1: ILC DRs main parameters [2]

\begin{tabular}{|l|c|c|}
\hline Bunch population & $N$ & $2 \times 10^{10}$ \\
Energy & & $5.0 \mathrm{GeV}$ \\
Ring circumference & $2 \pi R$ & $6695 \mathrm{~m}$ \\
Momentum compaction & $\alpha$ & $4.2 \times 10^{-4}$ \\
Synchrotron tune & $\nu_{s}$ & 0.064 \\
Rms bunch length & $\sigma_{z 0}$ & $9.0 \mathrm{~mm}$ \\
Rms energy spread & $\sigma_{\delta 0}$ & $1.28 \times 10^{-3}$ \\
Long. damping time & & $12.9 \mathrm{~ms}$ \\
RF voltage & & $24 \mathrm{MV}$ \\
RF frequency & & $650 \mathrm{MHz}$ \\
Number of cavities & & 18 \\
Wiggler straights & & $300 \mathrm{~m}$ \\
\hline
\end{tabular}

the study was entirely based on use of simple analytical models for the impedance of the various machine components. For example, the contribution from mostly inductive vacuum components like tapers, BPMs, etc. was modelled using the impedance $Z(\omega)=-i \omega(L / c) /(1-i \omega a / c)^{3 / 2}$, where $\omega$ is frequency and $L$ and $a$ are parameters. For each lattice under consideration $L$ and $a$ were set by simple scaling laws (accounting for parameters like the pipe radius, number of BPMs, etc.) starting from the inductance $L$ and loss factor $\kappa$ (related to $a$ ) for small vacuum components measured in an existing machine (PEP-II LER). This impedance model, combined with that for the resistive wall and RF cavities, was then used to calculate

$$
[\mathrm{Z} / \mathrm{n}]=\frac{\sigma_{z 0}}{R} \sum_{n=-\infty}^{\infty}\left|\frac{Z\left(n \omega_{0}\right)}{n}\right| e^{-\left(n \sigma_{z 0} \omega_{0} / c\right)^{2}},
$$

( $\omega_{0}$ is revolution frequency) taken as a figure of merit to gauge the sensitivity of the lattices to single-bunch instabilities. The values found for $[\mathrm{Z} / \mathrm{n}]$ spanned the range between 200 and $700 \mathrm{~m} \Omega$ with that of OCS, the predecessor of the current baseline lattice, estimated at $[\mathrm{Z} / \mathrm{n}]$ $=465 \mathrm{~m} \Omega$. These values were generally larger than $[\mathrm{Z} / \mathrm{n}]_{\mathrm{th}}=\sqrt{\pi / 2} Z_{0} \gamma \alpha \sigma_{\delta 0}^{2} \sigma_{z 0} / r_{e} N(\gamma$ is Lorentz energy factor, $r_{e}$ is electron radius) indicated as the critical value for instability by the Boussard criterion [8], which is often referred to for quick estimates. For the OCS lattice $[\mathrm{Z} / \mathrm{n}]_{\text {th }}=134 \mathrm{~m} \Omega$. One should be aware, however, that the Boussard criterion has a restricted range of applicability and can be quite inaccurate-generally overestimating the impedance effect-outside this range. Indeed for the PEP-II LER, $[\mathrm{Z} / \mathrm{n}]_{\text {th }}$ is almost half of $[\mathrm{Z} / \mathrm{n}]$ estimated using the above prescription, while there is no experimental evidence of any instability up to several multiples of the design LER bunch population.

D04 Instabilities - Processes, Impedances, Countermeasures 


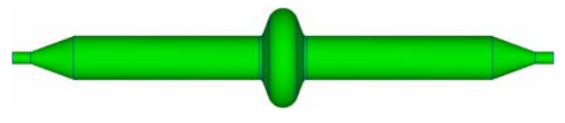

Figure 1: Sketch of RF cavity with tapers.

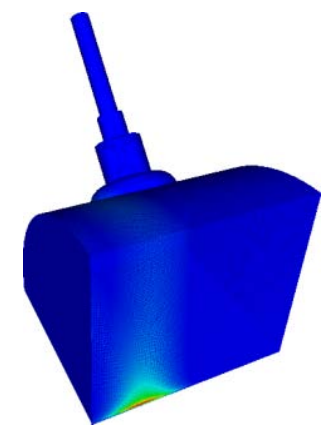

Figure 2: Section of the BPM model used for the wakepotential calculations.

\section{IMPEDANCE MODELLING}

A program to carry out systematic impedance modelling of individual machine components was initiated following the choice of the baseline DR lattice.

In most cases, because of the non-trivial geometries involved analytical modelling is inadequate and one needs to rely on extensive numerical simulations. For geometries with azimuthal symmetry a $2 \mathrm{D}$ approximation is generally adequate and standard 2D time-domain wakefield codes such as ABCI are suitable for this purpose. For more complicated devices 3-dimensional modelling, as implemented for example in codes like MAFIA, is required. For the 3D work documented in this paper, we employed a recently developed parallel finite-element time-domain code, T3P, which can conform to the boundaries of complex geometry, and is capable of treating very large systems using multiple processor computing [4].

Ideally, for beam dynamics studies one would like to have access to the wakefield Green function. Unfortunately this cannot be determined numerically as discretization of the problem sets an upper bound to the frequency spectrum making it impossible to resolve a delta-function like driving bunch. Instead a pseudo Green function is determined

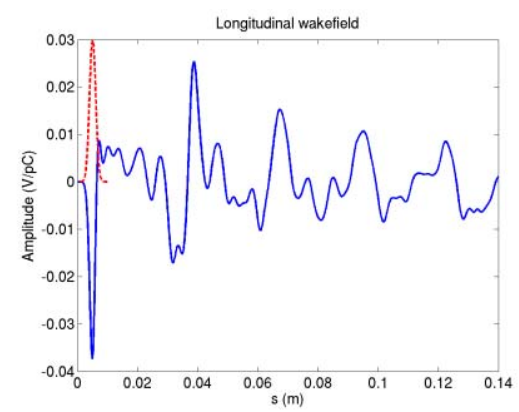

Figure 3: Wake potential from a single BPM

05 Beam Dynamics and Electromagnetic Fields

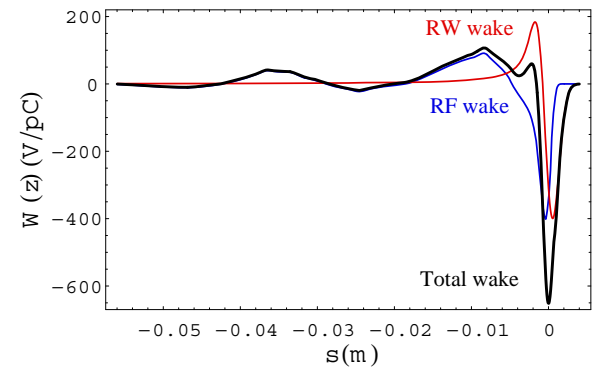

Figure 4: Wake potential for resistive wall (red curve), the $18 \mathrm{RF}$ cavities (blue curve), and their sum (black curve).

by computing the fields generated by a driving bunch with a small but finite length. Use of pseudo Green functions for tracking is an adequate approximation provided that the length of the driving bunch be sufficiently small compared to the length of the bunches in the DRs. In our calculations we stayed focused on short-range wakefields, relevant for single bunch dynamics.

We started by directing our efforts toward evaluating the impedances of those components that are believed to contribute significantly to the impedance budget, like the rf cavities and BPMs. Additional devices will be included at a later stage. As already mentioned, lacking the technical design of the damping ring components our strategy at present is to adopt the design of components in existing machines and scale them to meet the DRs specifications when necessary.

The ILC DRs will be equipped with 18 superconducting cavities at $650 \mathrm{MHz}$, a convenient harmonic of the 1.3 $\mathrm{GHz}$ frequency of the main linac rf structures. Examples of installed superconduncting rf cavities are at KEKB and at Cornell CESR. Because both sets of cavities operate in the neighborhood of $500 \mathrm{MHz}$ frequency and have tapers connecting to the ring beampipe with aperture different from that of the DRs, their design is not immediately usable for our purposes and would have to be scaled to match both the $2.5 \mathrm{~cm}$ DR pipe radius at the end of the tapers and the design frequency. The $2 \mathrm{D}$ longitudinal wakefield calculation for a $0.5 \mathrm{~mm}$ gaussian driving bunch shown as a blue curve in Fig. 4 was derived from scaled versions of the Cornell cavities (Fig. 1) and includes the effect of the tapers.

For the BPMs, we took as a model the design of the devices installed in PEP-II. The PEP-II BPMs have four buttons of diameter $1.5 \mathrm{~cm}$ located on the wall of a $4.8 \mathrm{~cm}$ radius beampipe (Fig. 2). In our adaptations to the DRs we scaled the button diameter to $0.8 \mathrm{~cm}$. So far we have produced 3D calculations of wakefields driven by a $1 \mathrm{~mm}$ bunch (Fig. 3). Excitation of resonances with long time effects can be seen in the picture. The long time tail of the wake is not of immediate concern for single bunch effects but it should be carefully investigated in future optimization of the BPM design as it could affect the coupled-bunch instabilities.

D04 Instabilities - Processes, Impedances, Countermeasures 


\section{INSTABILITY THRESHOLDS}

Given a model of short-range wakefield a number of methods are available for studying single-bunch beam dynamics. These include macroparticle simulations, direct Vlasov solvers, linear analysis using mode decomposition or solutions of the linearized Vlasov equation in the time domain. In preparation for the ILC-DR activities we carried out benchmarking [3] of some of the tools we have developed, which we intend to use in our studies. An example of this effort is shown in Fig. 5 reporting the current thresholds (normalized) for a simple resonator model of impedance [5] calculated using macroparticle simulations, which include radiation effects [6], and a direct Vlasov solver [7]. A comparison with a Boussard-like estimate is also shown.

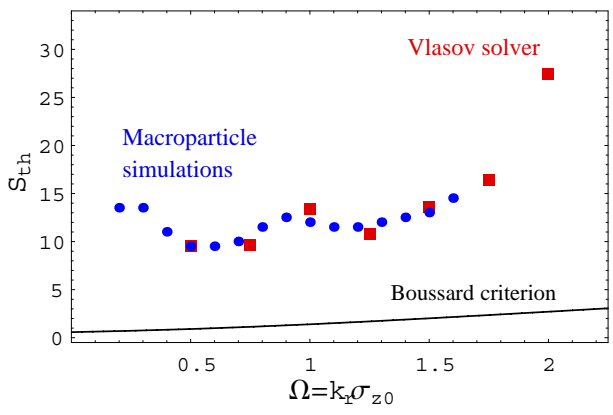

Figure 5: Instability thresholds (normalized, [5]) for a broadband resonator with $Q=1$.

We report a preliminary estimate of instability threshold based on the wake potentials for the RF cavities and the resistive wall effects - which are significant sources of the machine impedance. For the RF cavities we used the model discussed in the previous section.

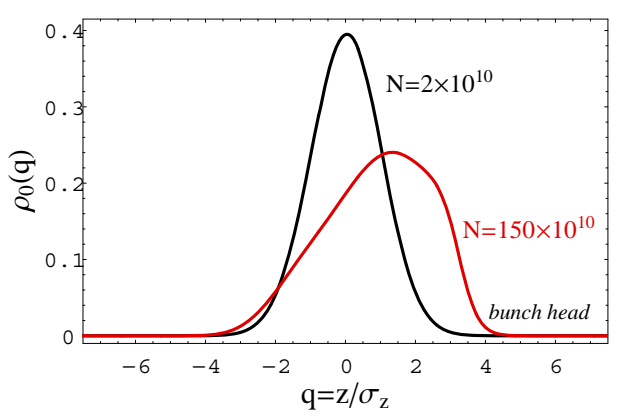

Figure 6: Haïssinski equilibria for nominal bunch population and just below instability threshold.

For the resistive wall effects, we made use of an analytical formula [8] valid for an infinitely long, straight vacuum chamber with circular cross section of radius $b$. However we did not use the reported expression for the wake potential directly but the result of its convolution with a 1 $\mathrm{mm}$ gaussian bunch. We separated the contribution from the (about $300 \mathrm{~m}$ long) wiggler sections and the rest of the 05 Beam Dynamics and Electromagnetic Fields

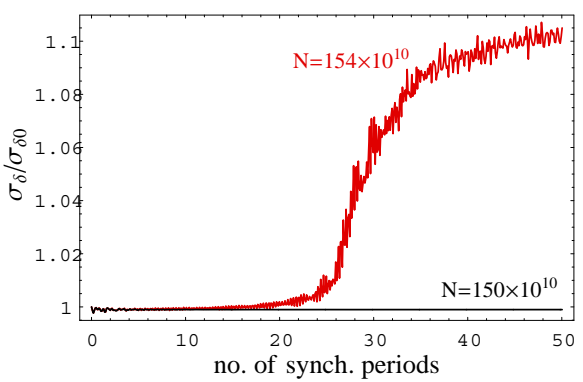

Figure 7: Evolution of energy spread slightly above and below instability starting from a Haïssinski equilibrium.

machine, as the pipe radius according to current specifications is slightly smaller ( $b=2.3$ vs. $b=2.5 \mathrm{~cm}$.). The Al conductivity $\left(\sigma=3.5 \times 10^{7} \Omega^{-1} \mathrm{~m}^{-1}\right)$ was assumed for the pipe walls. The resulting wake used for our estimates is shown as the black curve in Fig. 4. The instability was determined by looking for variations in the beam energy spread starting from a slightly perturbed Haïssinski distribution and neglecting radiation damping. The results of our calculations, obtained using a direct Vlasov solver, place the threshold well above the design bunch population at around $N=1.5 \times 10^{12}$. The Boussard criterion with the prescription of Eq. (1) yields $N=1.1 \times 10^{11}$. By comparison, our current estimate for the OCS lattice considered in [1] using the same wake potential model is about $N=4.3 \times 10^{11}$ - the main difference in this older lattice being in a smaller bunch length $\sigma_{z 0}=6 \mathrm{~mm}$ and momentum compaction $\alpha=1.6 \times 10^{-4}$. While abundantly above the nominal bunch population we should emphasize that these estimates are for a preliminary and still largely incomplete model of the machine impedance and meaningful conclusions should wait until a more comprehensive impedance model will be used in our estimates.

\section{REFERENCES}

[1] A. Wolski et al. Edts, "Configuration Studies and Recommendations for the ILC Damping Rings", LBNL-59449 (2006); S. Heifets et al. SLAC-PUB-12128.

[2] G. Aarons et al., "International Linear Collider Reference Design Report”, ILC-REPORT-2007-01 (2007), available at http://www.linearcollider.org

[3] ILC-DR06 Workshop, Cornell U., (2006). Talks available at https://www.lepp.cornell.edu/ilc/pub/Public/DampingRings

[4] A. Candel et. al, "Parallel Higher-Order Finite Element method for Accurate Field Computations in Wakefield and PIC Simulations", ICAP06 (2006), to be published.

[5] K. Oide and K. Yokoya, KEK Preprint 90-10 (1990).

[6] S. Heifets and K. Bane, unpublished.

[7] M. Venturini, et al., Phys. Rev. ST Accel. Beams 8, 014202 (2005); A. Novokhatski, SLAC-PUB-11251 (2005).

[8] A. Chao, "Physics of Collective Beam Instabilities in High Energy Accelerators", John Wiley \& Sons, Inc., New York (1993).

D04 Instabilities - Processes, Impedances, Countermeasures 